Pacific Journal of Mathematics

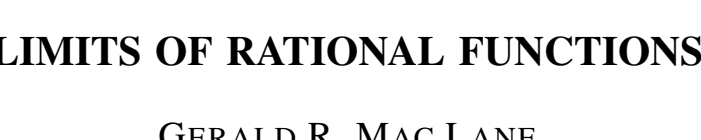




\title{
LIMITS OF RATIONAL FUNCTIONS
}

\author{
Gerald R. MaC Lane
}

Numerous results relating the location of the zeros of a sequence of polynomials to the form of possible limit functions of the sequence are known. These results are due in the main to Laguerre, Lindwart, Pólya, and Korevaar. Summaries and references are to be found in [5] and [1]. For example, the following is a theorem of Lindwart and Pólya [3]. Let $P_{n}(z)$ be a sequence of polynomials with all zeros real and let $P_{n}(z)$ converge uniformly in some domain to a function no identically zero. Then $P_{n}(z)$ converges, uniformly in every compact subset of the plane, to an entire function of the form $e^{-c z^{2}} f(z)$, where $c$ is a constant $\geqq 0$ and $f(z)$ is of genus $\leqq 1$.

We shall show, in Theorems 2 and 3, that the state of affairs is violently altered if instead of polynomials we consider rational functions with real zeros and real poles. Essentially, the convergence and-or non-convergence can be anything compatible with the fact that no limiting function, not identically zero, can have a non-real zero.

Various theorems of Saxer, Montel, and Obrechkoff specify the possible form of the limit of a sequence of rational functions. A résumé and references are contained in Obrechkoff [5]. All of these results depend on conditions on the rational functions involving either the location of the poles relative to the zeros or the behavior of expressions involving poles and residues.

The proof of Theorems 2 and 3 hinges on the fact that if $f(z)$ is holomorphic and $\neq 0$ in $\mathscr{I}_{z}>0$, then there exists a sequence of rational functions $R_{n}(z)$ with real zeros and poles such that $R_{n}(z) \rightarrow f(z)$ uniformly in every compact subset of $\mathscr{J} \boldsymbol{z}>0$. This is a special case of Theorem 1 below, which is similar to a previous result of ours for polynomials [4].

THEOREM 1. Let $\Gamma$ be a rectifiable Jordan curve on the z-sphere and let $D$ be one of the two domains determined by $\Gamma$. Let $f(z)$ be holomorphic and $\neq 0$ in $D$. Then there exists a sequence of rational functions $R_{n}(z), n \geqq 1$, such that all zeros and poles of $R_{n}(z)$ are on $\Gamma$ and $R_{n}(z) \rightarrow f(z)$ uniformly in every compact subset of $D$.

Note. If $\infty \notin \Gamma$, then each $R_{n}(z)$ is of the form $P(z) / Q(z)$, where $P$ and $Q$ are of the same degree and have zeros only on $\Gamma$. For $\infty \in \Gamma$, $P$ and $Q$ may have different degrees.

Received August 11, 1954. This research was supported by the United States Air Force, through the Office of Scientific Research of the Air Research and Development Command. 
Proof. If necessary, a linear transformation in $z$ enables us to assume that $D \cup \Gamma$ is bounded and $0 \in D$. As for the function $f(z)$ we may assume that $f(z)$ is holomorphic and $\neq 0$ in $D \cup \Gamma$. For let $P_{n}(z)$ be a sequence of polynomials tending to $\log f(z)$ uniformly in every compact subset of $D$. The sequence $\exp \left(P_{n}(z)\right)$ approaches $f(z)$ uniformly in every compact subset of $D$. Finally, we assume that $f(0)=1$.

The proof hinges on the following integral representation.

Lemma 1. Let $f(z)$ be holomorphic and $\neq 0$ in $D \cup \Gamma$. Then there exists a real-valued function $Q(\zeta)$, defined and continuous on $\Gamma$, such that

$$
\log f(z)=\int_{F} \frac{z}{\zeta(\zeta-z)} Q(\zeta) d \zeta
$$

Here $\log f(z)$ is that branch which vanishes at $z=0$.

The reality of $Q$ is essential in the use we shall make of this representation. We omit the proof of this lemma; it follows from [4, Lemma 2.3] after a simple integration by parts.

Now (1) may be written as

$$
\log f(z)=\int_{\Gamma}\left[\frac{1}{\zeta-z}-\frac{1}{\zeta}\right] Q(\zeta) d \zeta=c+\int_{\Gamma} \frac{Q(\zeta) d \zeta}{\zeta-z}, \quad z \in D,
$$

where $c$ is a constant. From the definition of the integral in (2) it follows that for each $\varepsilon>0$ and each compact subset $A \subset D$ there exists a set of points on $\Gamma, \zeta_{0}, \zeta_{1}, \cdots, \zeta_{m}=\zeta_{0}$, progressing around $\Gamma$ in the positive sense with respect to $D$ and such that

$$
\left|\log f(z)-c-\sum_{k=1}^{m} \frac{Q\left(\xi_{k}\right)}{\xi_{k}-z}\left(\zeta_{k}-\zeta_{k-1}\right)\right|<\varepsilon,
$$

where $\xi_{k}$ is any point of the interval $\left(\zeta_{k-1}, \zeta_{k}\right)$ of $\Gamma$. Since the values $Q\left(\xi_{k}\right)$ are real, Theorem 1 is a consequence of (3) and the following lemma.

Lemma 2. Let $\Gamma_{1}$ be any Jordan arc joining the distinct points $\zeta_{1}$ and $\zeta_{2}$. Then there exists a point $\xi$ on $\Gamma_{1}$ with the following property. For each real number $s$ there exists a sequence of rational functions $R_{m}(z)$, with all zeros and poles on $\Gamma_{1}$, such that

$$
R_{m}(z) \rightarrow \exp \left[\frac{s\left(\zeta_{2}-\zeta_{1}\right)}{\xi-z}\right]
$$


uniformly for $|z-\xi| \geqq \eta>0$.

Proof of Lemma 2. For each positive integer $m$ there exist two distinct points $\xi_{m}^{\prime}$ and $\xi_{m}^{\prime \prime}$ on $\Gamma_{1}$ such that the line through these points is parallel to the line through $\zeta_{1}$ and $\zeta_{2}$, and $\left|\xi_{m}^{\prime}-\xi_{m}^{\prime \prime}\right|<1 / m$. With proper labelling we may set

$$
\xi_{m}^{\prime \prime}-\xi_{m}^{\prime}=\delta_{m} e^{i \alpha}, \quad 0<\delta_{m}<1 / m,
$$

and

$$
\zeta_{2}-\zeta_{1}=b e^{i \alpha}
$$

The points $\xi_{m}^{\prime}, \xi_{m}^{\prime \prime}$ have at least one limit point on $\Gamma_{1}$, say $\xi$; clearly we may assume that $\xi_{m}^{\prime} \rightarrow \xi$ and $\xi_{m}^{\prime \prime} \rightarrow \xi$ as $m \rightarrow \infty$.

Set $\lambda_{m}=\left[b s / \delta_{m}\right]$ and

$$
R_{m}(z)=\left(\frac{z-\xi_{m}^{\prime \prime}}{z-\xi_{m}^{\prime}}\right)^{\lambda}
$$

where $[E]$ denotes the greatest integer not exceeding $E$. If $w=1 /(\xi-z)$, then

$$
\begin{aligned}
& z-\xi_{m}^{\prime \prime} \\
& z-\xi_{m}^{\prime}
\end{aligned}=1+\left(\xi_{m}^{\prime \prime}-\xi_{m}^{\prime}\right) w+\frac{\left(\xi_{m}^{\prime \prime}-\xi_{m}^{\prime}\right)\left(\xi-\xi_{m}^{\prime}\right) w^{2}}{1-\left(\xi-\xi_{m}^{\prime}\right) w}
$$

For $|z-\xi| \geqq \eta>0, w$ is bounded and

$$
\begin{aligned}
\log R_{m}(z) & =\lambda_{m}\left(\xi_{m}^{\prime \prime}-\xi_{m}^{\prime}\right) w+o\left(\lambda_{m}\left|\xi_{m}^{\prime \prime}-\xi_{m}^{\prime}\right|\right) \\
& =\frac{s\left(\zeta_{2}-\zeta_{1}\right)}{\xi-z}+o(1),
\end{aligned}
$$$$
m \rightarrow \infty,
$$

uniformly for $|z-\xi| \geq \eta>0$. Exponentiating yields (4). This completes the proof of Theorem 1 .

THEOREM 2. Let $D$ be a simply-connected domain contained in $\mathscr{I}_{z}>0$ and let $D^{*}$ be the reflection of $D$ in $\mathscr{I}_{z=0}$. Let $f(z)$ be an arbitrary holomorphic function in $D, f \neq 0$ in $D$. Let $f *(z)=\overline{f(\bar{z})} ; f^{*}(z)$ is holomorphic and $\neq 0$ in $D^{k}$. Then there exists a sequence of rational functions $R_{n}(z)$ such that

$1^{\circ}$ All the zeros and poles of $R_{n}(z)$ are real.

$2^{\circ} \quad R_{n}(z) \rightarrow f(z)$ uniformly in every compact subset of $D$ and $R_{n}(z) \rightarrow$ $c f^{*}(z)$ uniformly in every compact subset of $D^{*}$, where $c$ is a constant of modulus 1 .

$3^{\circ}$ The sequence $R_{n}(z)$ is non-normal at every point of the complement of $D \cup D^{*}$. 
Proof. By use of Runge's theorem it is easy to show, by approximating simultaneously to two different functions, that there exists a sequence of polynomials $P_{n}(z)$ with the properties:

(a) $P_{n}(z) \rightarrow \log f(z)$ uniformly in every compact subset of $D$.

(b) For any $z_{0} \notin D$ and any $r>0$ there exists $n_{0}$ such that for $n>$ $n_{0}$ the values assumed by $w=P_{n}(z)$ for $\left|z-z_{0}\right|<r$ include points of $\mathscr{R} w<-n$ as well as points of $\mathscr{R} w>n$.

By Theorem 1 there exists a sequence of rational functions $R_{n}(z)$ with real zeros and poles such that

$$
\left|R_{n}(z)-e^{P_{n}(z)}\right|<\frac{1}{n}, \quad z \in\{|z| \leqq n\} \cap\{\mathscr{J} z \geqq 1 / n\} .
$$

The first part of $2^{\circ}$ follows from (5) and (a). From (5) and (b) it follows that the sequence $R_{n}(z)$ is non-normal at every point of $\left\{\mathscr{J}_{z \geq 0} \geq-\right.$ $D$.

Since $R_{n}(z)$ has real zeros and poles we have

$$
R_{n}(\bar{z})=e^{i \alpha_{n}} \overline{R_{n}(z)},
$$

where $\alpha_{n}$ is a sequence of real constants. The remaining conclusions of Theorem 2 follow provided we pick a subsequence of the $R_{n}$ such that $\alpha_{n} \rightarrow \alpha$.

It is possible to replace the $f(z)$ of Theorem 2 by the constant zero. Namely, if $R_{n}(z)$ is the sequence obtained from Theorem 2 when $f(z) \equiv$ 1 , then $R_{n}(z) / n$ will tend to zero in $D \cup D^{*}$ and the specifications of (b) are strong enough so that this new sequence is still non-normal outside of $D \cup D^{*}$.

THEOREM 3. Let $D$ be a simply-connected domain in the z-plane, symmetric with respect to the real axis and containing no points of the interval $x \leqq 0$ of the real axis. Let $f(z)$ be holomorphic and $\neq 0$ in $D$, and let $f(x)$ be real on the single interval $\gamma$ common to $D$ and the real axis. Then there exists a sequence of rational functions $R_{n}(z)$ such that

$1^{\circ}$ All zeros and poles of $R_{n}(z)$ are real and negative.

$2^{\circ} \quad R_{n}(z) \rightarrow f(z)$ uniformly in every compact subset of $D$.

$3^{\circ}$ The sequence $R_{n}(z)$ is non-normal at every point of the complement of $\mathrm{D}$.

Proof. Since it is permissible to change the signs of $f(z)$ and $R_{n}(z)$, we may assume that $f(x)>0$ on $\gamma$. Now $\zeta=\xi+i \eta=z^{1 / 2}$ maps the $z$ plane, cut along the negative real axis onto $\xi>0$ and maps $D$ onto $\Delta$, a domain symmetric with respect to $\eta=0$ and containing a single interval $\Gamma$ of $\eta=0$. Set $f(z)=F(\zeta)$. 
By using Runge's theorem again it is easily seen that there exists a sequence of entire functions $g_{n}(\zeta)=\exp P_{n}(\zeta)$ such that:

(6a) $g_{n}(\zeta) \neq 0, g_{n}(\xi)$ is real and positive.

(6b) $g_{n}(\zeta) \rightarrow F(\zeta)$ uniformly in every compact subset of $\Delta$.

(6c) The sequence $g_{n}(\zeta)$ is non-normal at every point of the complement of $\Delta$.

Set

$$
h_{n}(\zeta)=\left(g_{n}(\zeta)\right)^{1 / 2},
$$

By virtue of $(6 a), h_{n}(\zeta)$ is entire. Also

$$
g_{n}(\zeta)=h_{n}^{2}(\zeta)=h_{n}(\zeta) \overline{h_{n}(\bar{\zeta})} .
$$

Let $A_{n}$ denote the compact set $\{\xi \geqq 1 / n\} \cap\{|\zeta| \leqq n\}$ and let

$$
M_{n}=1+\sup _{\zeta \in A_{n}}\left|h_{n}(\zeta)\right| \text {. }
$$

By Theorem 1 there exists a sequence of rational functions $r_{n}(\zeta)$ with all zeros and poles on $\xi=0$ such that

$$
\left|r_{n}(\zeta)-h_{n}(\zeta)\right|<1 / 3 n M_{n},
$$

Then $\left|\overline{r_{n}(\bar{\zeta})}-\overline{h_{n}(\bar{\zeta})}\right|$ has the same bound in $A_{n}$ and it follows that

$$
\left|r_{n}(\zeta) \overline{r_{n}(\bar{\zeta})}-h_{n}(\zeta) \overline{h_{n}(\overline{\bar{\zeta}})}\right|<1 / n,
$$

Combining (6), (7), and (8) it is clear that the function $R_{n}(z)$ defined by $R_{n}(z)=r_{n}(\zeta) \overline{r_{n}(\bar{\zeta})}$ has the properties $2^{\circ}$ and $3^{\circ}$ of Theorem 3 . Finally, each $r(\zeta)$ is of the form

$$
r(\zeta)=c \Pi_{j}\left(\zeta-i \alpha_{j}\right) / \prod_{k}\left(\zeta-i \beta_{k}\right),
$$

with $\alpha_{j}$ and $\beta_{k}$ real, and hence each $R(z)$ is of the form

$$
R(z)=|c|^{2} \prod_{j}\left(z+\alpha_{j}^{2}\right) / \prod_{k}\left(z+\beta_{k}^{2}\right) .
$$

Thus $R_{n}(z)$ also satisfies $1^{\circ}$ and the proof is complete.

\section{REFERENCES}

1. J. Korevaar, Approximation and interpolation applied to entire functions, thesis, Amsterdam, 1949.

2. - The zeros of approximating polynomials and the canonical representation of an entire function, Duke Math. J., 18 (1951), 573-592.

3. E. Lindwart und G. Pólya, Über einen Zusammenhang zwischen der Konvergenz von 
Polynomfolgen und der Verteilung ihrer Wurzeln, Rend. Cir. Mat. Palermo, 37 (1914), 297-304.

4. G.R. Mac Lane, Polynomials with zeros on a rectifiable Jordan curve, Duke Math. J., 16 (1949), 461-477.

5. N. Obrechkoff, Quelques classes de fonctions entières limites de polynomes et de fonctions méromorphes limites de fractions rationelles, Actualités Sci. Ind., 891, Paris, 1941.

The Rice Institute 


\section{PACIFIC JOURNAL OF MATHEMATICS}

\section{EDITORS}

H. L. Royden

Stanford University

Stanford, California

E. HewitT

University of Washington

Seattle 5 , Washington
R. P. Dilworth

California Institute of Technology Pasadena 4, California

E. G. Straus

University of California

Los Angeles 24, California

\section{ASSOCIATE EDITORS}

E. F. BECKENBACH

C. E. BURGESS

H. BUSEMANN

H. FEDERER

\author{
M. HALL \\ P. R. HALMOS \\ V. GANAPATHY IYER \\ R. D. JAMES
}

M. S. KNEBELMAN

I. NIVEN

T. G. OSTROM

M. M. SCHIFFER
J. J. STOKER

G. SZEKERES

F. WOLF

K. YOSIDA

\section{SUPPORTING INSTITUTIONS}

UNIVERSITY OF BRITISH COLUMBIA

CALIFORNIA INSTITUTE OF TECHNOLOGY

UNIVERSITY OF CALIFORNIA

MONTANA STATE UNIVERSITY

UNIVERSITY OF NEVADA

OREGON STATE COLLEGE

UNIVERSITY OF OREGON

UNIVERSITY OF SOUTHERN CALIFORNIA
STANFORD UNIVERSITY

UNIVERSITY OF UTAH

WASHINGTON STATE COLLEGE

UNIVERSITY OF WASHINGTON

AMERICAN MATHEMATICAL SOCIETY CALIFORNIA RESEARCH CORPORATION HUGHES AIRCRAFT COMPANY 


\section{Pacific Journal of Mathematics}

\section{Vol. 6, No. $1 \quad$ November, 1956}

David Blackwell, An analog of the minimax theorem for vector payoffs..... 1

L. W. Cohen, A non-archimedian measure in the space of real

sequences ..................................... 9

George Bernard Dantzig, Constructive proof of the Min-Max theorem ..... 25

Jim Douglas, On the numerical integration of quasilinear parabolic

differential equations ............................... 35

James Michael Gardner Fell, A note on abstract measure ............. 43

Isidore Isaac Hirschman, Jr., A note on orthogonal systems . . . . . . . . . . 47

Frank Harary, On the number of dissimilar line-subgraphs of a given

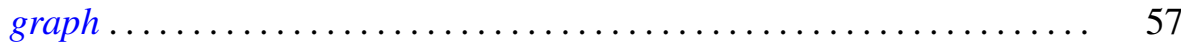

Newton Seymour Hawley, Complex bundles with Abelian group......... 65

Alan Jerome Hoffman, Morris Newman, Ernst Gabor Straus and Olga

Taussky, On the number of absolute points of a correlation ...........

Ernst Gabor Straus and Olga Taussky, Remark on the preceding paper.

Algebraic equations satisfied by roots of natural numbers . . ........ 97

Ralph D. James, Summable trigonometric series ................. 99

Gerald R. Mac Lane, Limits of rational functions . . . . . . . . . . . . . . . 111

F. Oberhettinger, Note on the Lerch zeta function ................. 117

Gerald C. Preston, On locally compact totally disconnected Abelian groups and their character groups ........................... 121

Vikramaditya Singh and W. J. Thron, On the number of singular points, located on the unit circle, of certain functions represented by

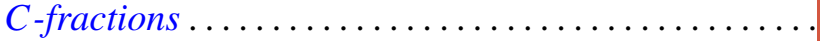

Sherman K. Stein, The symmetry function in a convex body ... 145 Edwin Weiss, Boundedness in topological rings.............

Albert Leon Whiteman, A sum connected with the series for the partition

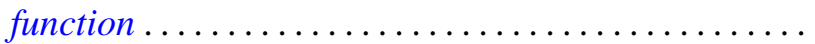

Alfred B. Willcox, Some structure theorems for a class of Banach algebras.

Joseph Lawrence Zemmer, Some remarks on p-rings and their Boolean geometry... 